\title{
Multidimensional Sinusoidal Frequency Estimation Using Subspace and Projection Separation Approaches
}

\author{
Longting Huang ${ }^{\ddagger}, \quad$ Yuntao $\mathrm{Wu}^{*}, \quad$ H. C. So ${ }^{\ddagger}, \quad$ Yanduo Zhang* and Lei Huang ${ }^{\dagger}$ \\ $\ddagger$ Department of Electronic Engineering, City University of Hong Kong, Hong Kong, China \\ * School of Computer Science and Engineering, Wuhan Institute of Technology, Wuhan, China \\ $\dagger$ Shenzhen Graduate School, Harbin Institute of Technology, Shenzhen, China
}

\begin{abstract}
In this correspondence, a computationally efficient method that combines the subspace and projection separation approaches is developed for $R$-dimensional ( $R$-D) frequency estimation of multiple sinusoids, where $R \geq 3$, in the presence of white Gaussian noise. Through extracting a 2-D slice matrix set from the multidimensional data, we devise a covariance matrix associated with one dimension, from which the corresponding frequencies are estimated using the root-MUSIC method. With the use of the frequency estimates in this dimension, a set of projection separation matrices is then constructed to separate all frequencies in the remaining dimensions. Root-MUSIC method is again applied to estimate these singletone frequencies while multidimensional frequency pairing is automatically attained. Moreover, the mean square error of the frequency estimator is derived and confirmed by computer simulations. It is shown that the proposed approach is superior to two state-of-the-art frequency estimators in terms of accuracy and computational complexity.
\end{abstract}

Index Terms

Multidimensional frequency estimation, subspace method, projection separation.

EDICS: SAS-STAT, DSP-TFSR, IMD-MDSP

\section{INTRODUCTION}

The topic of $R$-dimensional ( $R$-D) frequency estimation, where $R \geq 3$, has received extensive attention for its widespread applications in numerous fields such as MIMO wireless channel sounding [1], mobile communications [2], MIMO radar [3], sonar, seismology and nuclear magnetic resonance spectroscopy [4]. Many high-resolution subspace-based parameter estimation techniques have been proposed to solve this problem such as multidimensional folding (MDF) [1], unitary ESPRIT [5], improved MDF (IMDF) [6], MUSIC [7], rank reduction estimator (RARE) [8], decoupled root-MUSIC [9] and higher-order singular 
value decomposition (HOSVD) [10] methods. Unitary ESPRIT, MDF and IMDF techniques are based on the conventional ESPRIT approach where it is difficult to directly utilize the original multidimensional data. Typically, they require to enlarge the received data to construct a 2-D matrix with larger size, and then employ the ESPRIT-based method to obtain the desired frequency pairs. Consequently, their computational burden is very heavy particularly when the data size is large. The RARE algorithm vectorizes the observed data to exploit the Vandermonde structure and matrix polynomials are constructed to find the frequencies of each dimension. However, computing the polynomial coefficients is a highly demanding job. In the decoupled rootMUSIC algorithm, $R$-D harmonic retrieval is decomposed into $R$ 1-D problems by tensor decomposition [11], [12], which significantly reduces the computational load, but pairing of the $R$-D frequencies is required. On the other hand, the HOSVD method utilizes the structure inherent in the received data at the expense of a high computational complexity. In this work, our main contribution is to devise an accurate and computationally efficient estimator for multidimensional frequencies in the presence of white Gaussian noise with the use of the subspace and projection separation techniques.

The rest of this correspondence is organized as follows. The development of the proposed estimator is presented in Section II. There are two basic estimation steps as follows. We first extract a 2-D slice matrix set from the $R$-D signal to construct a covariance matrix associated with the first dimension, from which the corresponding frequencies are estimated using the root-MUSIC method. With the use of the frequency estimates in the first dimension, a set of projection separation matrices is then devised to separate all frequencies in the remaining dimensions. Root-MUSIC method is again utilized to find these singletone frequencies while multidimensional frequency pairing is automatically attained. In Section III, the computational complexity and mean square error (MSE) of the devised estimator are analyzed. Since our method exploits covariance matrices whose size is characterized by the number of sinusoids, its computational requirement is small when compared with the conventional schemes. Section IV includes numerical examples for validating the theoretical findings and evaluating the proposed approach by comparing with the IMDF [6] and HOSVD [10] methods as well as Cramér-Rao lower bound (CRLB). Finally, conclusions are drawn in Section V.

\section{Proposed Method}

\section{A. Signal Model}

The observed $R$-D signal model is

$$
x_{m_{1}, m_{2}, \cdots, m_{R}}=s_{m_{1}, m_{2}, \cdots, m_{R}}+q_{m_{1}, m_{2}, \cdots, m_{R}}, m_{r}=1,2, \cdots, M_{r}, r=1,2, \cdots, R
$$

where

$$
s_{m_{1}, m_{2}, \cdots, m_{R}}=\sum_{f=1}^{F} \alpha_{f} \prod_{r=1}^{R} e^{j \omega_{f, r} m_{r}}
$$

with $F$ being the number of sinusoids, which is assumed to be known a priori [13]. Here, $\alpha_{f}$ is the unknown complex amplitude of $f$ th tone, $\omega_{f, r} \in(-\pi, \pi)$ is the unknown frequency of $f$ th component in 
the $r$ th dimension. The $q_{m_{1}, m_{2}, \cdots, m_{R}}$ is a $R$-D circular complex white Gaussian noise with mean zero and unknown variance $\sigma_{q}^{2}$. It is assumed that $M_{r} \geq F, r=1,2, \cdots, R$, and the frequencies are distinct in at least one of the $R$ dimensions. Given the $M=\prod_{r=1}^{R} M_{r}$ samples of $x_{m_{1}, m_{2}, \cdots, m_{R}}$, our task is to find the $R F$ unknown frequency parameters, namely, $\left\{\omega_{f, r}\right\}, f=1,2, \cdots, F, r=1,2, \cdots, R$.

\section{B. Estimation in First Dimension}

For ease of presentation but without loss of generality, we start frequency estimation in the first dimension with the assumption that $\omega_{k, 1} \neq \omega_{l, 1}, k \neq l$. In tensor form, (1) is

$$
\mathcal{X}=\mathcal{S}+\mathcal{Q}
$$

where $\{\boldsymbol{\mathcal { X }}, \mathcal{S}, \mathcal{Q}\} \in \mathbb{C}^{M_{1} \times M_{2} \times \cdots \times M_{R}},[\mathcal{X}]_{m_{1}, m_{2}, \cdots, m_{R}}=x_{m_{1}, m_{2}, \cdots, m_{R}},[\mathcal{S}]_{m_{1}, m_{2}, \cdots, m_{R}}=s_{m_{1}, m_{2}, \cdots, m_{R}}$ and $[\mathcal{Q}]_{m_{1}, m_{2}, \cdots, m_{R}}=q_{m_{1}, m_{2}, \cdots, m_{R}}$. That is, $\mathcal{S}$ and $\mathcal{Q}$ are the noise-free and noise components of $\mathcal{X}$, respectively.

To reduce the dimension of $\mathcal{X}$, we define $\mathcal{X}_{r_{1}, r_{2}}$, which is a set of 2-D slice matrices extracting from $\mathcal{X}$, as follows:

$$
\mathcal{X}_{r_{1}, r_{2}}=\left\{\mathcal{X}\left(m_{1}, \cdots, m_{r_{1}-1},:, m_{r_{1}+1}, \cdots, m_{r_{2}-1},:, m_{r_{2}+1}, \cdots, m_{R}\right)\right\}
$$

where

$$
\begin{aligned}
& m_{r}=1,2, \cdots, M_{r}, \quad r=1,2, \cdots, R \quad \text { and } r \neq r_{1}, r_{2} \\
& {\left[\mathcal{X}\left(m_{1}, \cdots, m_{r_{1}-1},:, m_{r_{1}+1}, \cdots, m_{r_{2}-1},:, m_{r_{2}+1}, \cdots, m_{R}\right)\right]_{m_{r_{1}}, m_{r_{2}}}=x_{m_{1}, m_{2}, \cdots, m_{R}} .}
\end{aligned}
$$

Similarly, $\mathcal{S}_{1, r}$ is a noise-free 2-D matrix set extracting from $\mathcal{S}$ and each of the $\mathcal{S}\left(:, \cdots, m_{r-1}\right.$, : $\left., m_{r+1}, \cdots, m_{R}\right) \in \mathbb{C}^{M_{1} \times M_{r}}$ has the form of

$$
\left[\begin{array}{ccc}
\sum_{f=1}^{F} \alpha_{f} \prod_{\substack{n=2 \\
n \neq r}}^{R} e^{j \omega_{f, n} m_{n}} \cdot e^{j\left(\omega_{f, 1}+\omega_{f, r}\right)} & \ldots & \sum_{f=1}^{F} \alpha_{f} \prod_{\substack{n=2 \\
n \neq r}}^{R} e^{j \omega_{f, n} m_{n}} \cdot e^{j\left(\omega_{f, 1}+M_{r} \omega_{f, r}\right)} \\
\sum_{f=1}^{F} \alpha_{f} \prod_{\substack{n=2 \\
n \neq r}}^{R} e^{j \omega_{f, n} m_{n}} \cdot e^{j\left(2 \omega_{f, 1}+\omega_{f, r}\right)} & \ldots & \sum_{f=1}^{F} \alpha_{f} \prod_{\substack{n=2 \\
n \neq r}}^{R} e^{j \omega_{f, n} m_{n}} \cdot e^{j\left(2 \omega_{f, 1}+M_{r} \omega_{f, r}\right)} \\
\vdots & \ddots & \vdots \\
\sum_{f=1}^{F} \alpha_{f} \prod_{\substack{n=2 \\
n \neq r}}^{R} e^{j \omega_{f, n} m_{n}} \cdot e^{j\left(M_{1} \omega_{f, 1}+\omega_{f, r}\right)} & \cdots & \sum_{f=1}^{F} \alpha_{f} \prod_{\substack{n=2 \\
n \neq r}}^{R} e^{j \omega_{f, n} m_{n}} \cdot e^{j\left(M_{1} \omega_{f, 1}+M_{r} \omega_{f, r}\right)}
\end{array}\right] .
$$

It is easy to verify that $\mathcal{S}\left(:, \cdots, m_{r-1},:, m_{r+1}, \cdots, m_{R}\right)$ can be written as

$$
\mathcal{S}\left(:, \cdots, m_{r-1},:, m_{r+1}, \cdots, m_{R}\right)=\mathbf{G}_{1} \boldsymbol{\Gamma}_{1, r}\left(m_{2}, \cdots, m_{r-1}, m_{r+1}, \cdots, m_{R}\right) \mathbf{G}_{r}^{H}
$$


where

$$
\begin{aligned}
& \mathbf{G}_{r}=\left[\mathbf{g}_{1, r}, \mathbf{g}_{2, r}, \cdots, \mathbf{g}_{F, r}\right], r=1,2, \cdots, R \\
& \mathbf{g}_{f, r}=\left[a_{f, r}, a_{f, r}^{2}, \cdots a_{f, r}^{M_{r}}\right]^{T} \\
& a_{f, 1}=e^{j \omega_{f, 1}}, a_{f, r}=e^{-j \omega_{f, r}}, r=2,3, \cdots, R \\
& \boldsymbol{\Gamma}_{1, r}\left(m_{2}, \cdots, m_{r-1}, m_{r+1}, \cdots, m_{R}\right)=\operatorname{diag}\left(\alpha_{1} \prod_{\substack{n=2 \\
n \neq r}}^{R} a_{1, n}^{-m_{n}}, \alpha_{2} \prod_{\substack{n=2 \\
n \neq r}}^{R} a_{2, n}^{-m_{n}}, \cdots, \alpha_{F} \prod_{\substack{n=2 \\
n \neq r}}^{R} a_{F, n}^{-m_{n}}\right)
\end{aligned}
$$

with $(\cdot)^{H},(\cdot)^{T}$ and $\operatorname{diag}(\mathbf{a})$ being the conjugate transpose, transpose and diagonal matrix with vector a as its main diagonal, respectively.

Define $\hat{\mathbf{R}}_{1}$ as the sample covariance matrix for $\mathcal{X}_{1,2}$, which is computed as

$$
\hat{\mathbf{R}}_{1}=\frac{M_{1} M_{2}}{M} \sum_{m_{3}=1}^{M_{3}} \sum_{m_{4}=1}^{M_{4}} \cdots \sum_{m_{R}=1}^{M_{R}} \mathcal{X}\left(:,:, m_{3}, \cdots, m_{R}\right) \mathcal{X}^{H}\left(:,:, m_{3}, \cdots, m_{R}\right) .
$$

According to the structure of the slice matrix $\mathcal{X}\left(:,:, m_{3}, \cdots, m_{R}\right)$, the expected value of $\hat{\mathbf{R}}_{1}$, denoted by $\mathbf{R}_{1}$, is

$$
\begin{aligned}
\mathbf{R}_{1} & =\frac{M_{1} M_{2}}{M} \sum_{m_{3}=1}^{M_{3}} \cdots \sum_{m_{R}=1}^{M_{R}} \mathbf{G}_{1} \boldsymbol{\Gamma}_{1,2}\left(m_{3}, \cdots, m_{R}\right) \mathbf{G}_{2}^{H} \mathbf{G}_{2} \boldsymbol{\Gamma}_{1,2}^{H}\left(m_{3}, \cdots, m_{R}\right) \mathbf{G}_{1}^{H}+\sigma_{q}^{2} \mathbf{I}_{M_{1}} \\
& =\frac{M_{1} M_{2}}{M} \mathbf{G}_{1}\left(\sum_{m_{3}=1}^{M_{3}} \cdots \sum_{m_{R}=1}^{M_{R}} \boldsymbol{\Gamma}_{1,2}\left(m_{3}, \cdots, m_{R}\right) \mathbf{G}_{2}^{H} \mathbf{G}_{2} \boldsymbol{\Gamma}_{1,2}^{H}\left(m_{3}, \cdots, m_{R}\right)\right) \mathbf{G}_{1}^{H}+\sigma_{q}^{2} \mathbf{I}_{M_{1}} \\
& =\mathbf{G}_{1} \mathbf{B}_{1} \mathbf{G}_{1}^{H}+\sigma_{q}^{2} \mathbf{I}_{M_{1}}
\end{aligned}
$$

where

$$
\mathbf{B}_{1}=\frac{M_{1} M_{2}}{M} \sum_{m_{3}=1}^{M_{3}} \cdots \sum_{m_{R}=1}^{M_{R}} \boldsymbol{\Gamma}_{1,2}\left(m_{3}, \cdots, m_{R}\right) \mathbf{G}_{2}^{H} \mathbf{G}_{2} \boldsymbol{\Gamma}_{1,2}^{H}\left(m_{3}, \cdots, m_{R}\right) \in \mathbb{C}^{F \times F}
$$

and $\mathbf{I}_{M_{1}}$ is the $M_{1} \times M_{1}$ identity matrix.

In order to circumvent the problem of degraded estimation performance in case of closely-spaced frequencies in the same dimension, we further propose the forward-backward (FB) smoothing [14], [15] covariance matrix of $\hat{\mathbf{R}}_{1}$ :

$$
\hat{\mathbf{R}}_{1}^{\mathrm{FB}}=\frac{1}{2}\left(\hat{\mathbf{R}}_{1}+\mathbf{J}_{M_{1}} \hat{\mathbf{R}}_{1}^{*} \mathbf{J}_{M_{1}}\right)
$$

where $\mathbf{J}_{M_{1}} \in \mathbb{C}^{M_{1} \times M_{1}}$ is the exchange matrix with ones on its antidiagnoal and zeros elsewhere.

The expected value of $\hat{\mathbf{R}}_{1}^{\mathrm{FB}}$ is

$$
\mathbf{R}_{1}^{\mathrm{FB}}=\frac{1}{2}\left(\mathbf{R}_{1}+\mathbf{J}_{M_{1}} \mathbf{R}_{1}^{*} \mathbf{J}_{M_{1}}\right)=\mathbf{G}_{1} \tilde{\mathbf{B}}_{1} \mathbf{G}_{1}^{H}+\sigma_{q}^{2} \mathbf{I}_{M_{1}}
$$

where

$$
\begin{aligned}
\tilde{\mathbf{B}}_{1} & =\frac{1}{2}\left(\mathbf{B}_{1}+\mathbf{D}_{1} \mathbf{B}_{1}^{*} \mathbf{D}_{1}\right) \\
\mathbf{D}_{1} & =\operatorname{diag}\left(e^{-j M_{1} \omega_{1,1}}, e^{-j M_{1} \omega_{2,1}}, \cdots, e^{-j M_{1} \omega_{F, 1}}\right)
\end{aligned}
$$


On the other hand, $\hat{\mathbf{R}}_{1}^{\mathrm{FB}}$ can be decomposed using eigenvalue decomposition (EVD) as

$$
\hat{\mathbf{R}}_{1}^{\mathrm{FB}}=\hat{\mathbf{V}}_{1 s} \hat{\mathbf{\Lambda}}_{1 s} \hat{\mathbf{V}}_{1 s}^{H}+\hat{\mathbf{V}}_{1 n} \hat{\mathbf{\Lambda}}_{1 n} \hat{\mathbf{V}}_{1 n}^{H}
$$

where the column vectors of $\hat{\mathbf{V}}_{1 s} \in \mathbb{C}^{M_{1} \times F}$ and $\hat{\mathbf{V}}_{1 n} \in \mathbb{C}^{M_{1} \times\left(M_{1}-F\right)}$ are the eigenvectors that span the signal and noise subspaces of $\hat{\mathbf{R}}_{1}$, respectively, with the associated eigenvalues being the diagonal elements of $\hat{\Lambda}_{1 s}$ and $\hat{\Lambda}_{1 n}$.

Let

$$
\hat{\mathbf{E}}_{1}=\mathbf{I}_{M_{1}}-\hat{\mathbf{V}}_{1 s} \hat{\mathbf{V}}_{1 s}^{H}
$$

Employing root-MUSIC method to estimate the frequencies based on $\hat{\mathbf{E}}_{1}$, a null-spectrum function is constructed as:

$$
f_{1}(z)=\beta_{1}^{T}\left(z^{-1}\right) \hat{\mathbf{E}}_{1} \beta_{1}(z)
$$

where $\beta_{1}(z)=\left[z, z^{2}, \cdots, z^{M_{1}}\right]^{T}$. The polynomial $f_{1}(z)$ has $2\left(M_{1}-1\right)$ roots and the first dimension frequency estimates $\left\{\hat{\omega}_{f, 1}\right\}$ are obtained from the $F$ largest-magnitude roots inside the unit circle according to $z=e^{j \omega}$.

\section{Estimation in Remaining Dimensions}

With the use of $\left\{\hat{\omega}_{f, 1}\right\}$, a set of projection matrices $\hat{\mathbf{P}}_{f}^{\perp}, f=1,2, \cdots, F$, is constructed to estimate all $F$ frequencies one by one [19] in each of the $r$ th dimension, $r=2,3, \cdots, R$. The $\hat{\mathbf{P}}_{f}^{\perp}$ is defined as

$$
\hat{\mathbf{P}}_{f}^{\perp}=\mathbf{I}_{M_{1}}-\mathbf{H}_{f}\left(\mathbf{H}_{f}^{H} \mathbf{H}_{f}\right)^{-1} \mathbf{H}_{f}^{H}, \quad f=1,2, \cdots, F
$$

where

$$
\begin{aligned}
& \mathbf{H}_{f}=\left[\mathbf{h}\left(\hat{\omega}_{1,1}\right), \cdots, \mathbf{h}\left(\hat{\omega}_{f-1,1}\right), \mathbf{h}\left(\hat{\omega}_{f+1,1}\right), \cdots, \mathbf{h}\left(\hat{\omega}_{F, 1}\right)\right], \\
& \mathbf{h}\left(\hat{\omega}_{k, 1}\right)=\left[\hat{z}_{k, 1}, \hat{z}_{k, 1}^{2}, \cdots, \hat{z}_{k, 1}^{M_{1}}\right]^{T}, \quad \hat{z}_{k, 1}=e^{j \hat{\omega}_{k, 1}} .
\end{aligned}
$$

Analogous to (4), we construct the 2-D slice matrices set $\mathcal{X}_{1, r}$ and then use the projection matrix $\hat{\mathbf{P}}_{f}^{\perp}$ on $\mathcal{X}\left(:, \cdots, m_{r-1},:, m_{r+1}, \cdots, m_{R}\right) \in \mathbb{C}^{M_{1} \times M_{r}}$ to obtain $\mathcal{X}_{f, r}\left(:, \cdots, m_{r-1},:, m_{r+1}, \cdots, m_{R}\right)$ which contains only the information of $\left\{\omega_{f, r}\right\}$ in the $r$ th dimension associated with $\hat{\omega}_{f, 1}$.

The $\boldsymbol{\mathcal { X }}_{f, r}\left(:, \cdots, m_{r-1},:, m_{r+1}, \cdots, m_{R}\right)$ is defined as

$$
\begin{aligned}
& \mathcal{X}_{f, r}\left(:, \cdots, m_{r-1},:, m_{r+1}, \cdots, m_{R}\right)=\hat{\mathbf{P}}_{f}^{\perp} \mathcal{X}\left(:, \cdots, m_{r-1},:, m_{r+1}, \cdots, m_{R}\right) \\
& =\hat{\mathbf{P}}_{f}^{\perp} \mathbf{G}_{1} \boldsymbol{\Gamma}_{1, r}\left(m_{2}, \cdots, m_{r-1}, m_{r+1}, \cdots, m_{R}\right) \mathbf{G}_{r}^{H}+\hat{\mathbf{P}}_{f}^{\perp} \mathcal{Q}\left(:, \cdots, m_{r-1},:, m_{r+1}, \cdots, m_{R}\right)
\end{aligned}
$$

where $r=2,3, \cdots, R, f=1,2, \cdots, F$.

Similar to (11), we define the covariance matrix $\hat{\mathbf{R}}_{f, r}$

$$
\hat{\mathbf{R}}_{f, r}=\frac{M_{1} M_{r}}{M} \sum_{m_{2}=1}^{M_{2}} \cdots \sum_{m_{r-1}=1}^{M_{r-1}} \sum_{m_{r+1}=1}^{M_{r+1}} \cdots \sum_{m_{R}=1}^{M_{R}} \mathcal{X}_{f, r}^{H}\left(:, \cdots, m_{r-1},:, m_{r+1}, \cdots, m_{R}\right) .
$$


where $r=2,3, \cdots, R, f=1,2, \cdots, F$.

The expected value of $\hat{\mathbf{R}}_{f, r}$ is calculated as

$$
\begin{aligned}
\mathbf{R}_{f, r}= & \frac{M_{1} M_{r}}{M} \sum_{m_{2}=1}^{M_{2}} \cdots \sum_{m_{r-1}=1}^{M_{r-1}} \sum_{m_{r+1}=1}^{M_{r+1}} \cdots \sum_{m_{R}=1}^{M_{R}} \mathbf{G}_{r} \boldsymbol{\Gamma}_{1, r}^{H}\left(m_{2}, \cdots, m_{r-1}, m_{r+1}, \cdots, m_{R}\right) . \\
& \mathbf{G}_{1}^{H} \mathbf{P}_{f}^{\perp} \mathbf{P}_{f}^{\perp} \mathbf{G}_{1} \boldsymbol{\Gamma}_{1, r}\left(m_{2}, \cdots, m_{r-1}, m_{r+1}, \cdots, m_{R}\right) \mathbf{G}_{r}^{H}+\sigma_{q}^{2} \mathbf{I}_{M_{r}} \\
= & \mathbf{G}_{r} \mathbf{B}_{f, r} \mathbf{G}_{r}^{H}+\sigma_{q}^{2} \mathbf{I}_{M_{r}}
\end{aligned}
$$

where

$$
\begin{aligned}
& \mathbf{B}_{f, r}=\frac{M_{1} M_{r}}{M} \sum_{m_{2}=1}^{M_{2}} \cdots \sum_{m_{r-1}=1}^{M_{r-1}} \sum_{m_{r+1}=1}^{M_{r+1}} \cdots \sum_{m_{R}=1}^{M_{R}} \boldsymbol{\Gamma}_{1, r}^{H}\left(m_{2}, \cdots, m_{r-1}, m_{r+1}, \cdots, m_{R}\right) . \\
& \mathbf{G}_{1}^{H} \mathbf{P}_{f}^{\perp} \mathbf{P}_{f}^{\perp} \mathbf{G}_{1} \boldsymbol{\Gamma}_{1, r}\left(m_{2}, \cdots, m_{r-1}, m_{r+1}, \cdots, m_{R}\right) \in \mathbb{C}^{F \times F}, \\
& r=2,3, \cdots, R, \quad f=1,2, \cdots, F
\end{aligned}
$$

and

$$
\mathbf{P}_{f}^{\perp} \mathbf{G}_{1}=\left[\mathbf{0}, \mathbf{0}, \cdots, \mathbf{P}_{f}^{\perp} \mathbf{g}_{f, 1}, \cdots, \mathbf{0}\right] .
$$

with $\mathbf{0}$ being the $M_{1} \times 1$ zero vector. Furthermore, the FB smoothing version of $\hat{\mathbf{R}}_{f, r}$ is

$$
\hat{\mathbf{R}}_{f, r}^{\mathrm{FB}}=\frac{1}{2}\left(\hat{\mathbf{R}}_{f, r}+\mathbf{J}_{M_{r}} \hat{\mathbf{R}}_{f, r}^{*} \mathbf{J}_{M_{r}}\right)
$$

The expected value of $\hat{\mathbf{R}}_{f, r}^{\mathrm{FB}}$ is

$$
\mathbf{R}_{f, r}^{\mathrm{FB}}=\frac{1}{2}\left(\mathbf{R}_{f, r}+\mathbf{J}_{M_{r}} \mathbf{R}_{f, r}^{*} \mathbf{J}_{M_{r}}\right)=\mathbf{G}_{r} \tilde{\mathbf{B}}_{f, r} \mathbf{G}_{r}^{H}+\sigma_{q}^{2} \mathbf{I}_{M_{r}}
$$

where

$$
\begin{aligned}
& \tilde{\mathbf{B}}_{f, r}=\frac{1}{2}\left(\mathbf{B}_{f, r}+\mathbf{D}_{r} \mathbf{B}_{f, r}^{*} \mathbf{D}_{r}\right) \\
& \mathbf{D}_{r}=\operatorname{diag}\left(e^{j M_{r} \omega_{1, r}}, e^{j M_{r} \omega_{2, r}}, \cdots, e^{j M_{r} \omega_{F, r}}\right) .
\end{aligned}
$$

Taking EVD on $\hat{\mathbf{R}}_{f, r}^{\mathrm{FB}}$ yields

$$
\hat{\mathbf{R}}_{f, r}^{\mathrm{FB}}=\hat{\mathbf{V}}_{f, r s} \hat{\boldsymbol{\Lambda}}_{f, r s} \hat{\mathbf{V}}_{f, r s}^{H}+\hat{\mathbf{V}}_{f, r n} \hat{\boldsymbol{\Lambda}}_{f, r n} \hat{\mathbf{V}}_{f, r n}^{H}, \quad r=2,3, \cdots, R, f=1,2, \cdots, F
$$

where the column vectors of $\hat{\mathbf{V}}_{f, r s} \in \mathbb{C}^{M_{r} \times 1}$ and $\hat{\mathbf{V}}_{f, r n} \in \mathbb{C}^{M_{r} \times\left(M_{r}-1\right)}$ are the eigenvectors that span the signal and noise subspaces of $\hat{\mathbf{R}}_{f, r}$, respectively, with the associated eigenvalues being the diagonal elements of $\hat{\boldsymbol{\Lambda}}_{f, r s}$ and $\hat{\boldsymbol{\Lambda}}_{f, r n}$.

Define $\hat{\mathbf{E}}_{f, r}$ of the form:

$$
\hat{\mathbf{E}}_{f, r}=\mathbf{I}_{M_{r}}-\hat{\mathbf{V}}_{f, r s} \hat{\mathbf{V}}_{f, r s}^{H}, \quad r=2,3, \cdots, R, f=1,2, \cdots, F
$$

We see that the root-MUSIC method can also be used to obtain the $f$ th component in the $r$ th dimension frequency, $\hat{\omega}_{f, r}$, which is automatically paired with $\hat{\omega}_{f, 1}$ with the use of $\hat{\mathbf{P}}_{f}^{\perp}$. The corresponding null-spectrum function is

$$
f_{f, r}(z)=\beta_{f, r}^{T}\left(z^{-1}\right) \hat{\mathbf{E}}_{f, r} \beta_{f, r}(z)
$$


where $\beta_{f, r}(z)=\left[z, z^{2}, \cdots, z^{M_{r}}\right]^{T}$. The polynomial $f_{f, r}(z)$ has $2\left(M_{r}-1\right)$ roots and the $r$ th dimension frequency of $f$ th component $\hat{\omega}_{f, r}$ is obtained from the largest-magnitude root inside the unit circle according to $z=e^{-j \omega}, r=2,3, \cdots, R, f=1,2, \cdots, F$.

To summarize, the steps in the overall estimation procedure are:

1) Compute $\hat{\mathbf{R}}_{1}^{\mathrm{FB}}$ in (14) and perform its EVD

2) Compute $\hat{\mathbf{E}}_{1}$ using (19) and use (20) to compute $\hat{\omega}_{f, 1}, f=1,2, \cdots, F$

3) Construct $\hat{\mathbf{P}}_{f}^{\perp}$ in (21) and compute $\hat{\mathbf{R}}_{f, r}^{\mathrm{FB}}$ using (29)

4) Take the EVD on $\hat{\mathbf{R}}_{f, r}^{\mathrm{FB}}$, compute $\hat{\mathbf{E}}_{f, r}$ according to (34) and use (35) to obtain $\hat{\omega}_{f, r}, r=2,3, \cdots, R$, $f=1,2, \cdots, F$

\section{Algorithm AnAlysis}

\section{A. Computational Complexity}

The computational complexity of the proposed root-MUSIC algorithm is studied and the results are provided in Table I. In summary, its complexity is $\mathcal{O}\left(M\left(M_{1}+F \sum_{r=2}^{R}\left(M_{1}+M_{r}\right)\right)\right)$. On the other hand, the computational requirement in the IMDF method [6] is about $\mathcal{O}\left(M^{3}\right)$ and that of the HOSVD algorithm [10] is at least $\mathcal{O}\left(\prod_{r=1}^{R}\left(M_{r}-L_{r}+1\right) L_{r}^{3}\right)$ where $L_{r}$ is the number of spatial smoothing subarrays in the $r$ th dimension, $r=1,2, \cdots, R$. Clearly, the proposed method is more computationally attractive than [6] and [10].

\section{B. Mean Square Error}

Analogous to (18) and (33), we define

$$
\begin{aligned}
& \mathbf{R}_{1}^{\mathrm{FB}}=\mathbf{V}_{1 s} \boldsymbol{\Lambda}_{1 s} \mathbf{V}_{1 s}^{H}+\sigma_{q}^{2} \mathbf{V}_{1 n} \mathbf{V}_{1 n}^{H} \\
& \mathbf{R}_{f, r}^{\mathrm{FB}}=\lambda_{f, r s} \mathbf{v}_{f, r s} \mathbf{v}_{f, r s}^{H}+\sigma_{q}^{2} \mathbf{V}_{f, r n} \mathbf{V}_{f, r n}^{H}
\end{aligned}
$$

where $\mathbf{V}_{1 s}=\left[\mathbf{v}_{1}, \mathbf{v}_{2}, \cdots, \mathbf{v}_{F}\right], \mathbf{V}_{1 n}=\left[\mathbf{v}_{F+1}, \mathbf{v}_{F+2}, \cdots, \mathbf{v}_{M_{1}}\right], \boldsymbol{\Lambda}_{1 s}=\left[\lambda_{1}, \lambda_{2}, \cdots, \lambda_{F}\right]$ and $\mathbf{V}_{f, r n}=$ $\left[\mathbf{v}_{f, r}^{(2)}, \mathbf{v}_{f, r}^{(3)}, \cdots, \mathbf{v}_{f, r}^{\left(M_{r}\right)}\right]$. Note that $\mathbf{v}_{f}, f=1,2, \cdots, F$, is the signal subspace eigenvector of the first dimension, $\lambda_{f}$ are the associated eigenvalue with $\mathbf{v}_{f}$ while $\mathbf{V}_{1 n}$ is the noise subspace of the first dimension. Meanwhile, $\mathbf{v}_{f, r s}$ and $\lambda_{f, r s}$ are the eigenvector and eigenvalue of the signal subspace of the $f$ th component in the $r$ th dimension frequency, respectively, and $\mathbf{V}_{f, r n}$ is the noise subspace corresponding to $\mathbf{v}_{f, r s}$.

Following [15], the MSEs of the first dimension frequency estimates are computed as

$$
\mathbb{E}\left\{\left(\hat{\omega}_{f, 1}-\omega_{f, 1}\right)^{2}\right\}=\frac{M_{1} M_{2}}{2 M} \frac{\sigma_{q}^{2} \sum_{f=1}^{F} \frac{\lambda_{f}}{\left(\lambda_{f}-\sigma_{q}^{2}\right)^{2}}\left|\mathbf{v}_{f}^{H} \mathbf{g}_{f, 1}\right|^{2}}{\mathbf{d}_{f, 1}^{H} \mathbf{V}_{1 n} \mathbf{V}_{1 n}^{H} \mathbf{d}_{f, 1}}
$$

where $\mathbf{d}_{f, 1}=\mathrm{d} \mathbf{g}_{f, 1} / \mathrm{d} \omega_{f, 1}$, and $\mathbb{E}$ is the expectation operator. 
On the other hand, the MSEs of the frequency estimates in the remaining dimensions are

$$
\mathbb{E}\left\{\left(\hat{\omega}_{f, r}-\omega_{f, r}\right)^{2}\right\}=\frac{M_{1} M_{r}}{2 M} \frac{\sigma_{q}^{2} \frac{\lambda_{f, r s}}{\left(\lambda_{f, r s}-\sigma_{q}^{2}\right)^{2}}\left|\mathbf{v}_{f, r s}^{H} \mathbf{g}_{f, r}\right|^{2}}{\mathbf{d}_{f, r}^{H} \mathbf{V}_{f, r n} \mathbf{V}_{f, r n}^{H} \mathbf{d}_{f, r}}
$$

where $\mathbf{d}_{f, r}=\mathrm{d} \mathbf{g}_{f, r} / \mathrm{d} \omega_{f, r}$.

\section{Simulation Results}

Computer simulations have been conducted to evaluate the frequency estimation performance of the proposed approach for multiple 3-D and 4-D sinusoids in the presence of white Gaussian noise. The algorithms without and with FB smoothing, denoted by root MUSIC and FB root MUSIC, are examined. The average MSE of the $r$ th dimension, denoted by $\mathrm{AMSE}_{r}$, is employed as the performance measure. All results provided are averaged from 200 independent runs. Apart from CRLB [6], [17]-[18], the performance of the proposed approach is compared with that of the IMDF [6] and HOSVD [10] algorithms. The signal power is defined as $\sigma_{s}^{2}=\left(\sum_{m_{1}=1}^{M_{1}} \cdots \sum_{m_{R}=1}^{M_{R}}\left|s_{m_{1}, m_{2}, \cdots, m_{R}}\right|^{2}\right) / M$ and $q_{m_{1}, m_{2}, \cdots, m_{R}}$ is scaled to produce different signal-to-noise ratio (SNR) where $\mathrm{SNR}=\sigma_{s}^{2} / \sigma_{q}^{2}$.

In the first test, we consider 3-D frequency estimation with $M_{1}=M_{2}=M_{3}=15$ and the number of tones is $F=3$. The sinusoidal parameters are $\left\{\alpha_{f}\right\}=\left\{1, e^{j 0.3 \pi}, e^{j 0.5 \pi}\right\},\left\{\omega_{1, r}\right\}=\{0.1 \pi, 0.25 \pi, 0.4 \pi\}$, $\left\{\omega_{2, r}\right\}=\{0.25 \pi, 0.4 \pi, 0.1 \pi\}$ and $\left\{\omega_{3, r}\right\}=\{0.4 \pi, 0.1 \pi, 0.25 \pi\}$. The results of $\mathrm{AMSE}_{r}$ versus SNR are plotted in Figures 1 to 3. It is observed that although the MSE of the proposed approach in the first dimension is comparable with that of HOSVD scheme, the former is superior to [6] and [10], with performance close to CRLB, in the remaining dimensions. The theoretical calculations of (38) and (39) also agree very well with the simulation results for sufficiently high SNR conditions. The above test is repeated with identical frequency scenario of $\left\{\omega_{1, r}\right\}=\{0.1 \pi, 0.3 \pi, 0.4 \pi\},\left\{\omega_{2, r}\right\}=\{0.25 \pi, 0.3 \pi, 0.4 \pi\}$ and $\left\{\omega_{3, r}\right\}=\{0.4 \pi, 0.35 \pi, 0.45 \pi\}$, and the results are plotted in Figures 4 to 6 . In this challenging case, the proposed method without FB smoothing performs the worst because there are identical frequencies in two dimensions. Nevertheless, the one with FB smoothing outperforms [6] and [10] for all dimensions and its performance attains CRLB in the second and third dimensions.

In the second experiment, the average computational time of the investigated algorithms versus different $M_{1}$ with $M_{1}=M_{2}=M_{3}$ in 3-D frequency estimation with $F=3$ are plotted in Figure 7. It is seen that the proposed approach is much more computationally efficient than the IMDF and HOSVD algorithms, which aligns with the complexity analysis in Section III.

In the third experiment, $\mathrm{AMSE}_{r}$ for different frequency separation by varying $\omega_{2,1}$ is studied. We consider two 3-D tones with $M_{1}=M_{2}=M_{3}=15$. The signal parameters are $\left\{\alpha_{f}\right\}=\left\{1, e^{j 0.3 \pi}\right\},\left\{\omega_{1, r}\right\}=$ $\{0.1 \pi, 0.25 \pi, 0.4 \pi\}, \omega_{2,2}=0.4 \pi$ and $\omega_{2,3}=0.1 \pi$ while $\omega_{2,1}$ is varying from $0.1 \pi$ to $0.6 \pi$. The corresponding results at SNR $=10 \mathrm{~dB}$ are shown in Figures 8 to 10 . It is observed that the estimation performance of the proposed approach is inferior to the IMDF and HOSVD schemes in the first dimension when the frequency separation is less than $0.1 \pi$. Nevertheless, its performance is comparable to them in the other two dimensions. 
Finally, 4-D frequency estimation is studied and the results of $\mathrm{AMSE}_{r}$ versus SNR are plotted in Figures 11 to 14 . The number of sinusoids is $F=3$ while $M_{1}=15, M_{2}=M_{3}=M_{4}=7$. The signal parameters are $\left\{\alpha_{f}\right\}=\left\{1, e^{j 0.3 \pi}, e^{j 0.5 \pi}\right\},\left\{\omega_{1, r}\right\}=\{0.1 \pi, 0.25 \pi, 0.4 \pi, 0.1 \pi\},\left\{\omega_{2, r}\right\}=\{0.25 \pi, 0.4 \pi, 0.4 \pi, 0.1 \pi\}$ and $\left\{\omega_{3, r}\right\}=\{0.4 \pi, 0.25 \pi, 0.45 \pi, 0.15 \pi\}$. Again, the superiority of the proposed approach over [6] and [10] is observed. Moreover, its MSE attains CRLB for the second to fourth dimensions at sufficiently high SNRs.

\section{CONCLUSION}

A new approach for frequency estimation of multidimensional sinusoids in additive white circular Gaussian noise has been developed. The main idea in our methodology is to rearrange the $R$-D sinusoids as a series of 2-D slice matrices and combine the subspace and projection separation techniques. The frequencies in one dimension are first estimated using the root-MUSIC algorithm, which are then utilized to separate the frequencies in the remaining dimensions. Using the separated data, the remaining frequencies are then estimated one by one using the root-MUSIC method such that the multidimensional parameters are automatically paired. It is shown that the proposed algorithm is superior to the IMDF and HOSVD methods in terms of computational load and estimation performance.

\section{ACKNOWLEDGMENT}

The work described in this paper was jointly supported by the grant from the National Natural Science Foundation of China (Project No.61172150) and the Research Plan Project of Hubei Provincial Department of Education (No. Q20091501).

\section{REFERENCES}

[1] K. N. Mokios, N. D. Sidiropoulos, M. Pesavento and C. F. Mecklenbräuker, "On 3-D harmonic retrieval for wireless channel sounding," Proc., IEEE International Conference on Acoustics, Speech and Signal Processing, vol. 2, pp. II89-II92, Montreal, Quebec, Canada, May 2004.

[2] X. Liu, N. D. Sidiropoulos and A. Swami, "Blind high-resolution localization and tracking of multiple frequency hopped signals," IEEE Trans. Signal Process., vol. 50, no. 4, pp. 889-901, Apr. 2002.

[3] D. Nion and N. D. Sidiropoulos, "Tensor algebra and multidimensional harmonic retrieval in signal processing for MIMO radar," IEEE Trans. Signal Process., vol. 58, no. 11, pp. 5693-5705, Nov. 2010.

[4] Y. Li, J. Razavilar and K. J. R. Liu, “A high-resolution technique for multidimensional NMR spectroscopy,” IEEE Transactions on Biomedical Engineering, vol. 45, no. 1, pp. 78-86, Jan. 1998.

[5] M. Haardt and J.A. Nossek, "Simultaneous schur decomposition of several nonsymmetric matrices to achieve automatic pairing in multidimensional harmonic retrieval problems," IEEE Trans. Signal Process., vol. 46, no. 1, pp. 161-169, Jan. 1998.

[6] J. Liu and X. Liu,"An eigenvector-based approach for multidimensional frequency estimation with improved identifiability," IEEE Trans. Signal Process., vol. 54, no. 12, pp. 4543-4556, Dec. 2006.

[7] H. L. Van Trees, Optimum Array Processing: Detection, Estimation, and Modulation Theory. New York: Wiley, 2002, pt. IV.

[8] M. Pesavento, C. F. Mecklenbräker and J. F. Böme, "Multidimensional rank reduction estimator for parametric MIMO channel models," EURASIP J. Appl. Signal Process., vol. 2004, pp. 1354-1363, Jan. 2004.

[9] R. Boyer, "Decoupled root-music algorithm for multidimensional harmonic retrieval," IEEE Workshop on Signal Processing Advances in Wireless Communications, pp. 16-20, Recife, Brazil, Jul. 2008. 
[10] M. Haardt, F. Roemer and G. Del Galdo, "Higher-order SVD-based subspace estimation to improve the parameter estimation accuracy in multidimensional harmonic retrieval problems," IEEE Trans. Signal Process., vol. 56, no. 7, pp. 3198-3213, Jul. 2008.

[11] L. de Lathauwer, B. de Moor and J. Vanderwalle, "A multilinear singular-value decomposition," SIAM J. Matrix Anal. Appl., vol. 21, no. 4, pp. 1253-1278, 2000.

[12] L. de Lathauwer, B. de Moor and J. Vanderwalle, "On the best rank-1 and rank- $\left(r_{1}, r_{2}, \cdots, r_{n}\right)$ approximation of higher-order tensors," SIAM J. Matrix Anal. Appl., vol. 21, no. 4, pp. 1324-1342, 2000.

[13] J. P. C. L Da Costa, F. Roemer, M. Haardt and R. T. de Sousa Jr, "Multidimensional model order selection,” EURASIP Journal on Advances in Signal Processing, 2011, 2011:26.

[14] D. A. Linebarger, R. D. DeGroat and E. M. Dowling, "Efficient direction-finding methods employing forward-backward averaging," IEEE Trans. Signal Process., vol. 42, no. 8, pp. 2136-2145, Aug. 1994.

[15] M. Pesavento, A.B. Gershman and M. Haardt, "Unitary root-MUSIC with a real-valued eigendecomposition: a theoretical and experimental performance study," IEEE Trans. Signal Process., vol. 48, no. 5, pp. 1306-1314, May 2000.

[16] Y.-Y. Wang, J.-T. Chen and W.-H. Fang, “TST-MUSIC for joint DOA-delay estimation,” IEEE Trans. Signal Process., vol. 46, pp. 721-729, Apr. 2001.

[17] R. Boyer, "Deterministic asymptotic Cramér-Rao lower bound for the multidimensional harmonic model," Signal Processing, vol. 88 , no. 12 , pp. 2869-2877, Dec. 2008.

[18] X. Liu and N.D. Sidiropoulos, "Cramér-Rao lower bound for low-rank decomposition of multidimensional arrays," IEEE Trans. Signal Process., vol. 49, no. 9, pp. 2074-2086, Sep. 2001.

[19] Y.-Y. Wang, J.-T. Chen and W.-H. Fang, "TST-MUSIC for joint DOA-delay estimation,” IEEE Trans. Signal Process., vol. 46, no. 3, pp. 721-729, Apr. 2001.

[20] A. Thakre, M. Haardt, F. Roemer and K. Giridhar, "Tensor-based spatial smoothing (TB-SS) using multiple snapshots," IEEE Trans. Signal Process., vol. 58, no. 5, pp.2715-2728, May 2010.

TABLE I: Computational complexity of FB root-MUSIC algorithm

\begin{tabular}{lll}
\hline Operation & Dimension & Multiplication \\
\hline Construct $\hat{\mathbf{R}}_{1}^{F B}$ & $M_{1} \times M_{1}$ & $M_{1} M$ \\
EVD of $\hat{\mathbf{R}}_{1}^{F B}$ & $M_{1} \times M_{1}$ & $25 M_{1}^{3}$ \\
Compute $\hat{\mathbf{E}}_{1}$ & $M_{1} \times M_{1}$ & $M_{1}^{2} F$ \\
Construct $\hat{\mathbf{R}}_{f, r}^{F B}$ & $M_{r} \times M_{r}$ & $F M\left(M_{1}+M_{r}\right)$ \\
EVD of $\hat{\mathbf{R}}_{f, r}^{F B}$ & $M_{r} \times M_{r}$ & $25 M_{r}^{3} F$ \\
Compute $\hat{\mathbf{E}}_{f, r}$ & $M_{r} \times M_{r}$ & $M_{r}^{2} F$ \\
Total & & $\mathcal{O}\left(M\left(M_{1}+F \sum_{r=2}^{R}\left(M_{1}+M_{r}\right)\right)\right)$ \\
\hline
\end{tabular}



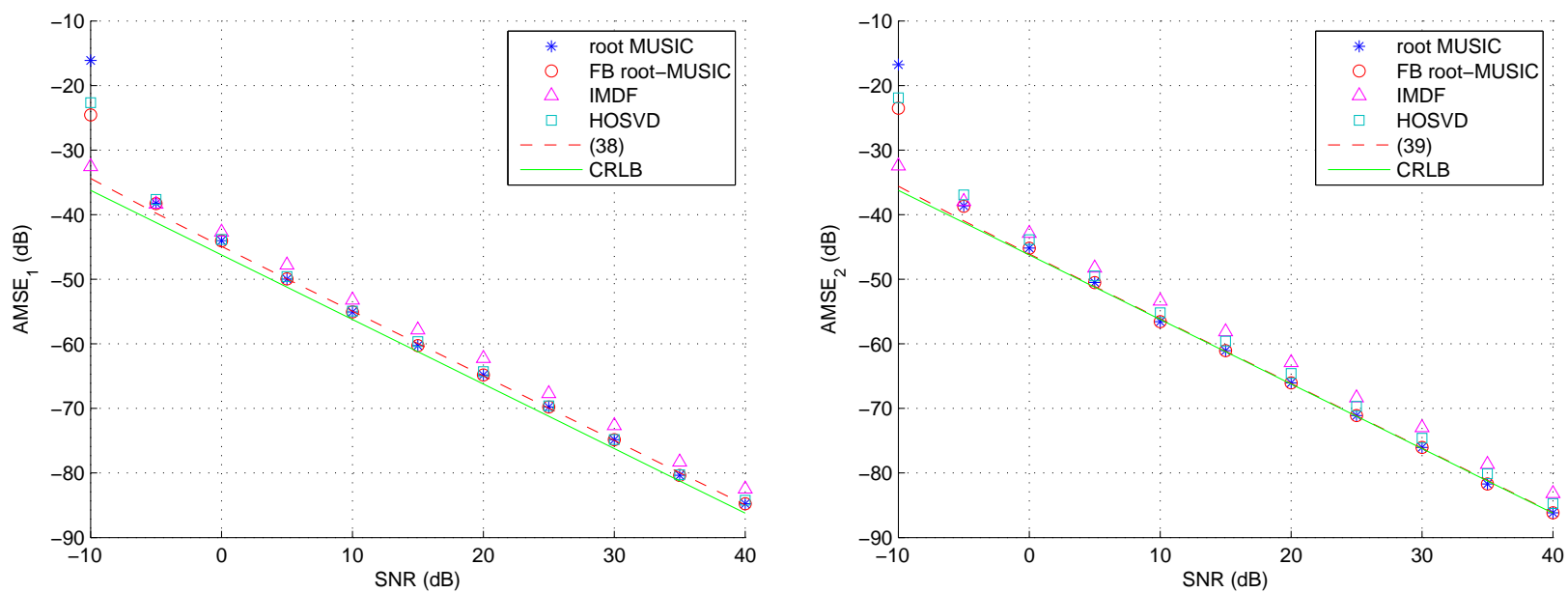

Fig. 1: $\mathrm{AMSE}_{1}$ versus SNR with distinct frequenciesFig. 2: $\mathrm{AMSE}_{2}$ versus SNR with distinct frequencies
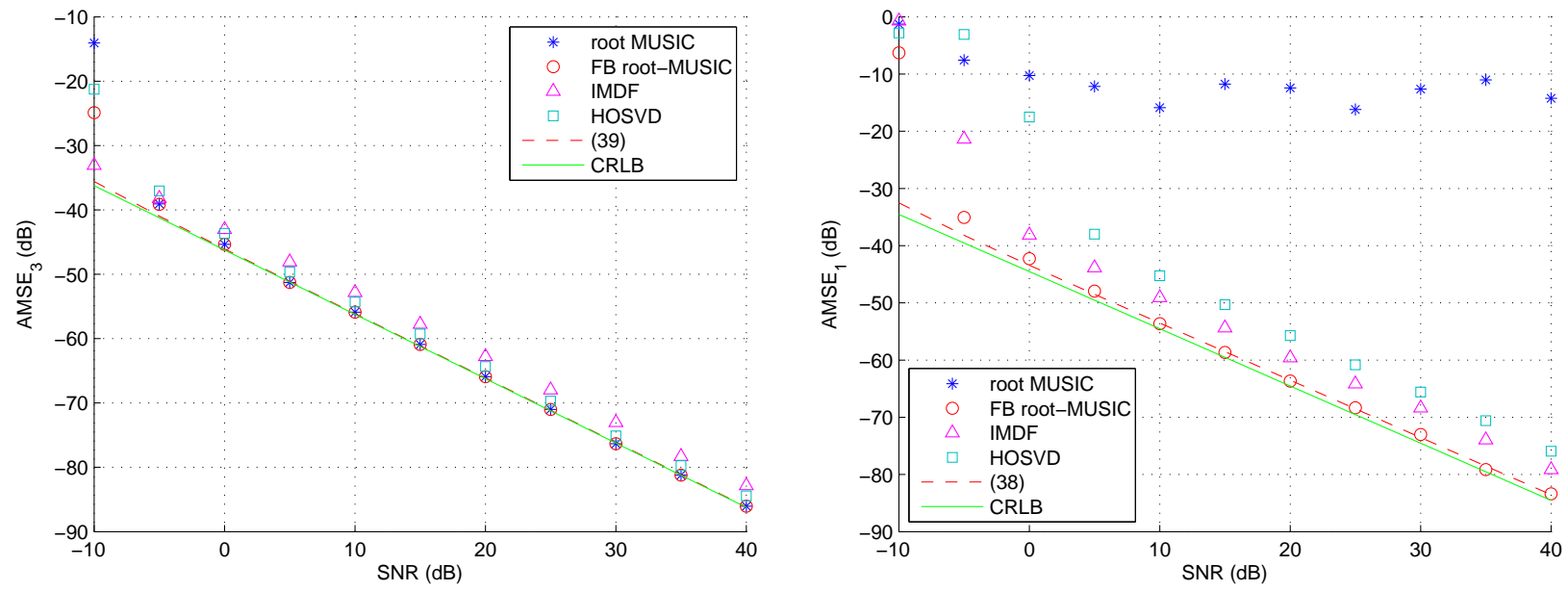

Fig. 3: $\mathrm{AMSE}_{3}$ versus SNR with distinct frequenciesFig. 4: $\mathrm{AMSE}_{1}$ versus SNR with identical frequencies
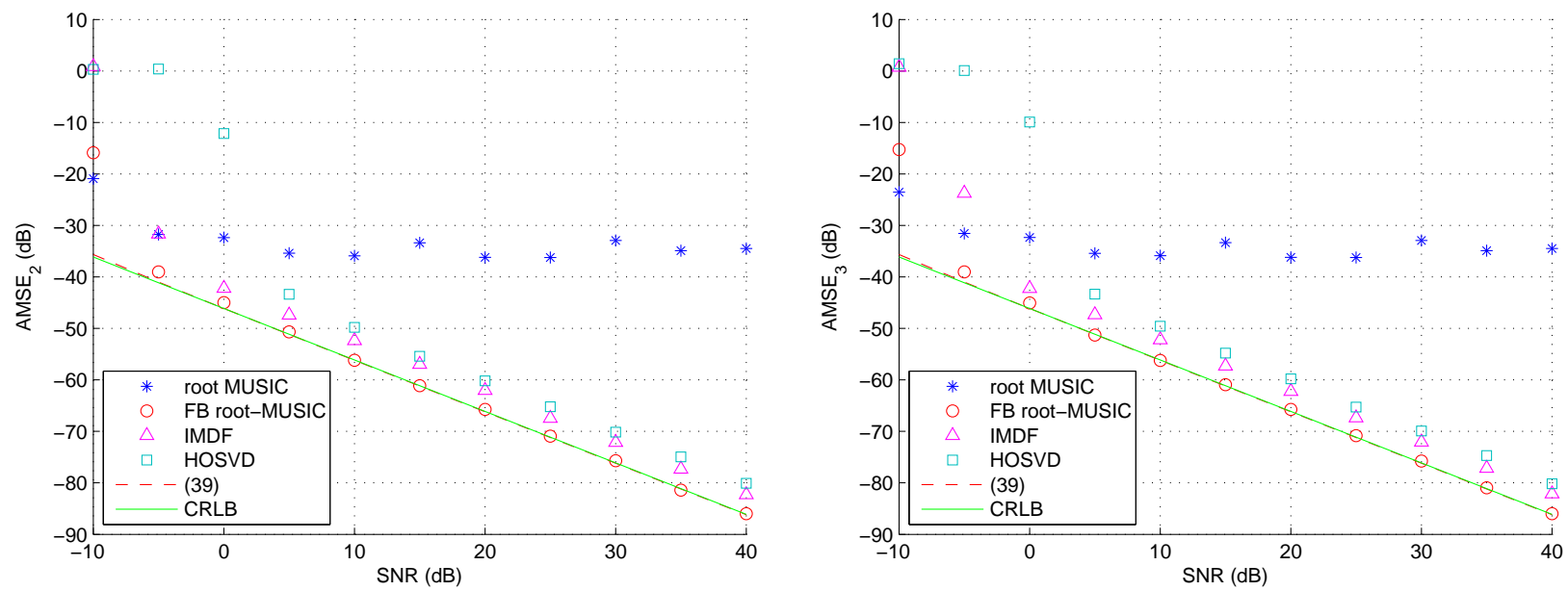

Fig. 5: $\mathrm{AMSE}_{2}$ versus SNR with identical frequenciesFig. 6: $\mathrm{AMSE}_{3}$ versus $\mathrm{SNR}$ with identical frequencies 


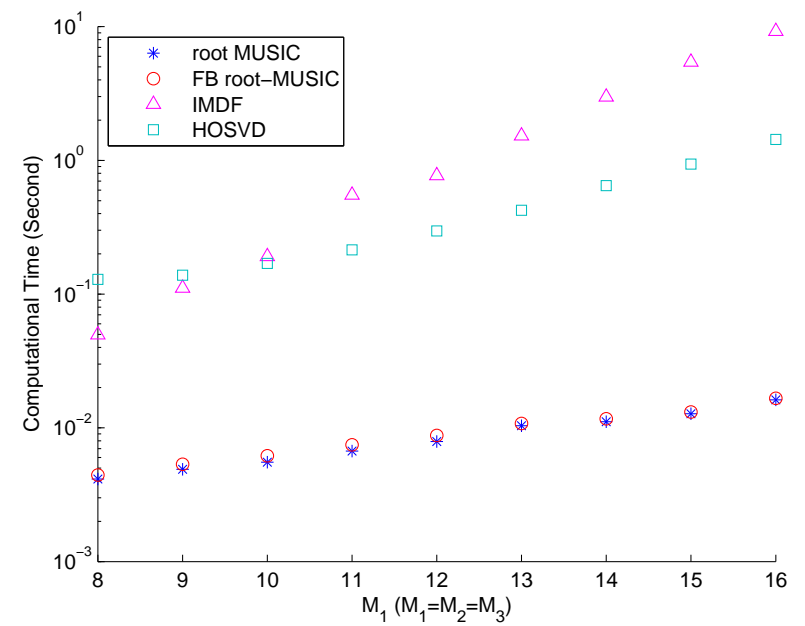

Fig. 7: Computational time versus $M_{1}$

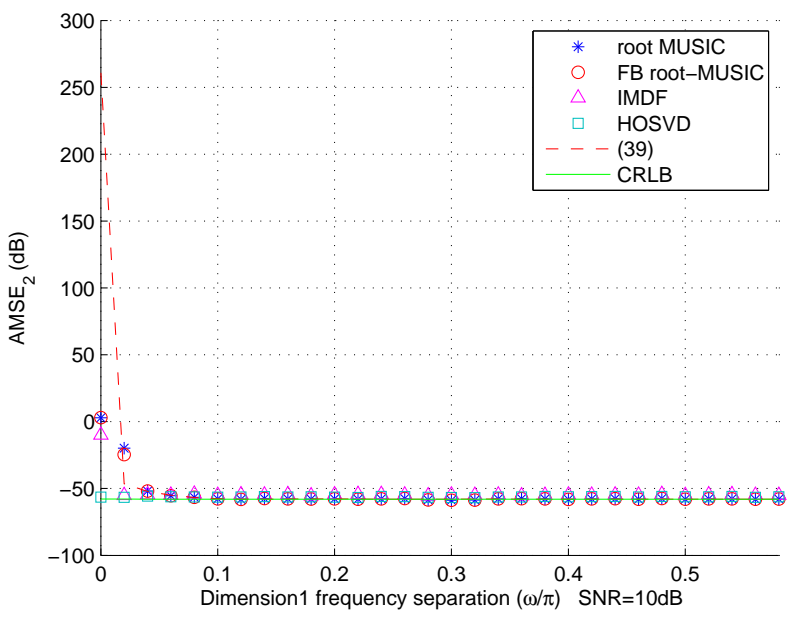

Fig. 9: $\mathrm{AMSE}_{2}$ versus frequency separation

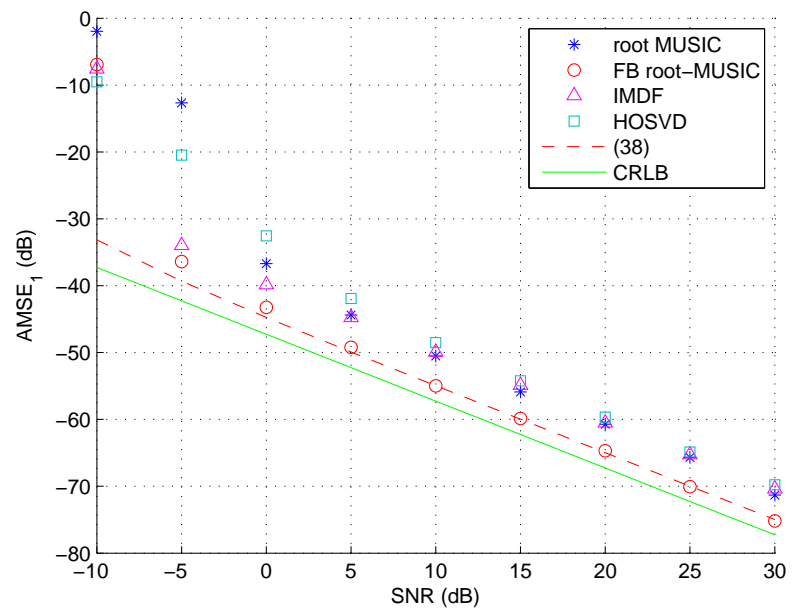

Fig. 11: $\mathrm{AMSE}_{1}$ versus SNR for $4 \mathrm{D}$ Data

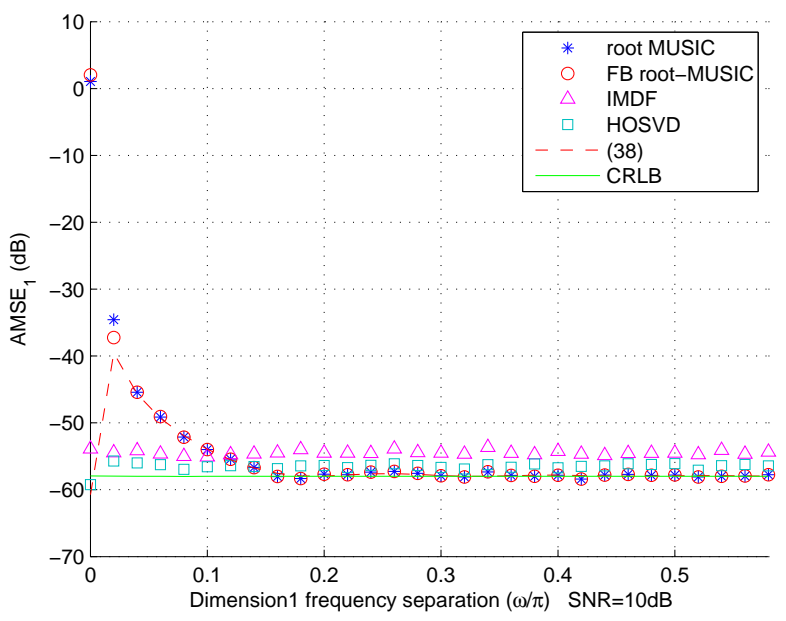

Fig. 8: $\mathrm{AMSE}_{1}$ versus frequency separation

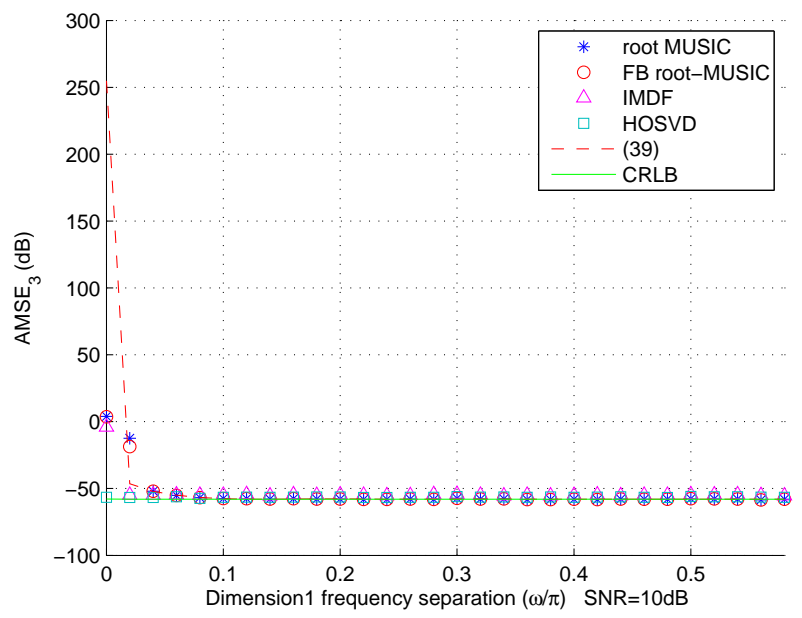

Fig. 10: $\mathrm{AMSE}_{3}$ versus frequency separation

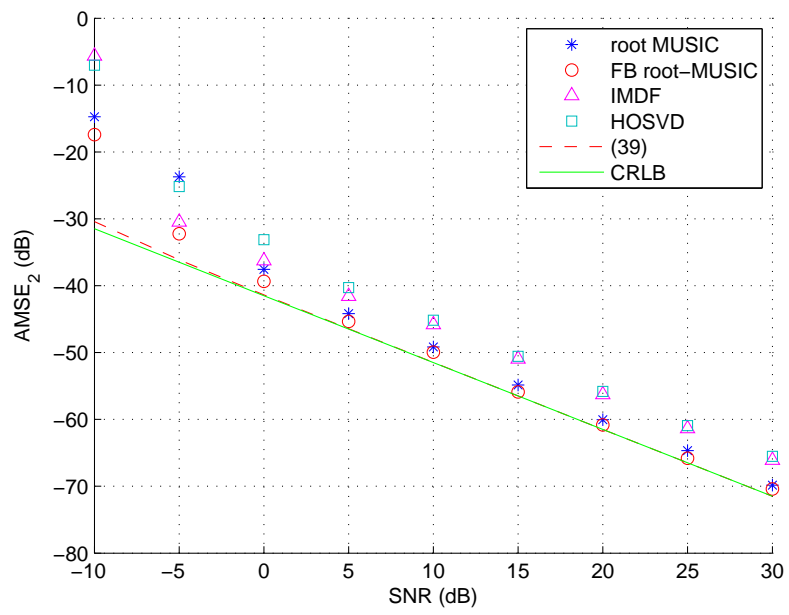

Fig. 12: $\mathrm{AMSE}_{2}$ versus SNR for 4D Data 


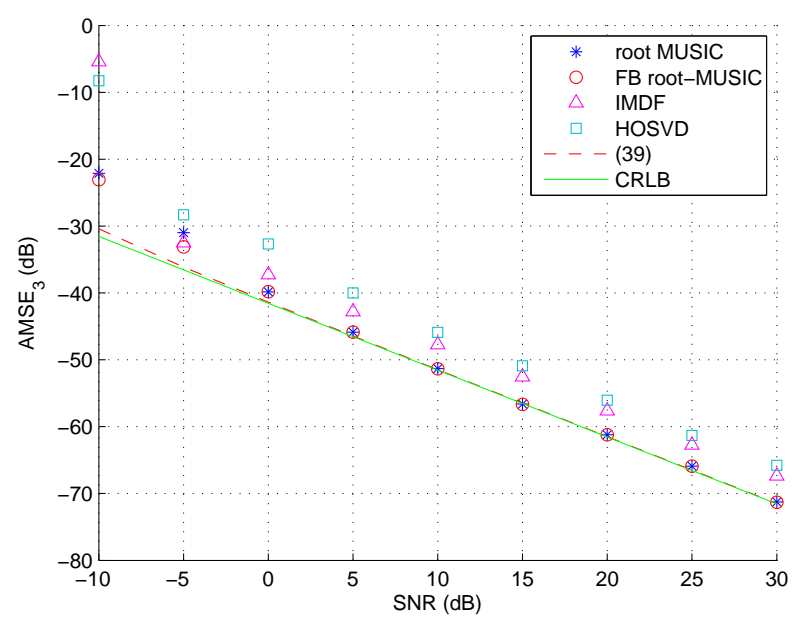

Fig. 13: $\mathrm{AMSE}_{3}$ versus SNR for 4D Data

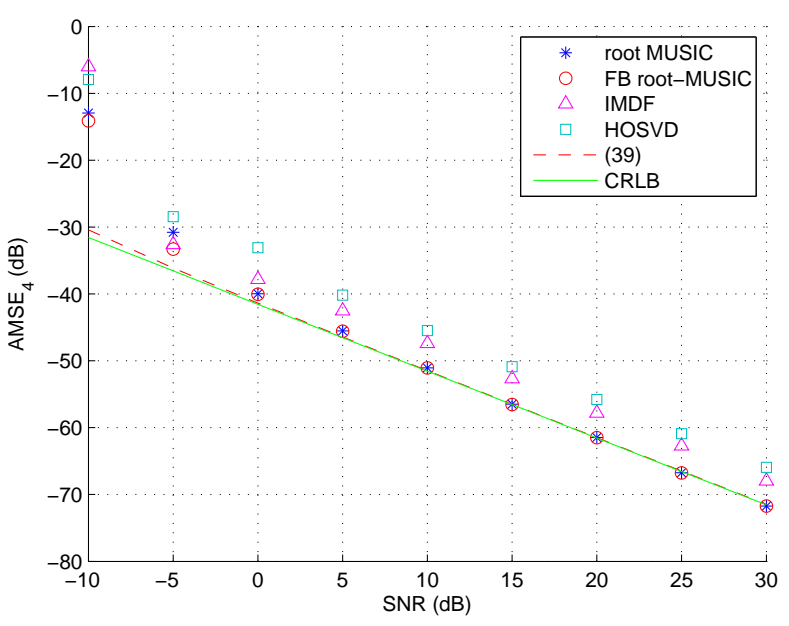

Fig. 14: $\mathrm{AMSE}_{4}$ versus SNR for 4D Data 